\title{
General Conditions for Universality of Quantum Hamiltonians
}

\author{
Tamara Kohler®, ${ }^{1, *}$ Stephen Piddock, ${ }^{2,3}$ Johannes Bausch, ${ }^{4}$ and Toby Cubitt ${ }^{1}$ \\ ${ }^{1}$ Department of Computer Science, University College London, London WC1E 6BT, United Kingdom \\ ${ }^{2}$ School of Mathematics, University of Bristol, Bristol BS8 1QU, United Kingdom \\ ${ }^{3}$ Heilbronn Institute for Mathematical Research, Bristol BS8 1UG, United Kingdom \\ ${ }^{4}$ Department of Applied Mathematics and Theoretical Physics, University of Cambridge, Cambridge CB2 ITN, \\ United Kingdom
}

(Received 19 March 2021; revised 10 November 2021; accepted 10 December 2021; published 13 January 2022; corrected 7 February 2022)

\begin{abstract}
Recent work has demonstrated the existence of universal Hamiltonians - simple spin-lattice models that can simulate any other quantum many-body system to any desired level of accuracy. Until now, proofs of universality have relied on explicit constructions, tailored to each specific family of universal Hamiltonians. In this work, we go beyond this approach and completely classify the simulation ability of quantum Hamiltonians by their complexity classes. We do this by deriving necessary and sufficient complexitytheoretic conditions characterizing universal quantum Hamiltonians. Although the result concerns the theory of analog Hamiltonian simulation - a promising application of near-term quantum technology - the proof relies on abstract complexity-theoretic concepts and the theory of quantum computation. As well as providing simplified proofs of previous Hamiltonian universality results and offering a route to new universal constructions, the results in this paper give insight into the origins of universality; for example, finally explaining the previously noted coincidences between families of universal Hamiltonian and classes of Hamiltonians appearing in complexity theory.
\end{abstract}

DOI: 10.1103/PRXQuantum.3.010308

\section{INTRODUCTION}

Recent work has precisely defined what it means for one quantum system to simulate the full physics of another [1] and has demonstrated that - within very demanding definitions of what it means for one system to simulate another - there exist families of local Hamiltonians that are universal, in the sense that they can simulate all other Hamiltonians to any accuracy desired. This rigorous mathematical framework of Hamiltonian simulation not only gives a theoretical foundation for describing analog Hamiltonian simulation - one of the most promising applications of quantum computing in the noisy intermediate-scale quantum (NISQ) era. It also unifies many previous Hamiltonian complexity results and implies new ones [1]. And it has even found applications in constructing the first rigorous holographic dualities between local Hamiltonians, providing toy models of anti-de Sitter/conformal field theory (AdS-CFT) duality in quantum gravity [2].

\footnotetext{
*tamara.kohler.16@ucl.ac.uk

Published by the American Physical Society under the terms of the Creative Commons Attribution 4.0 International license. Further distribution of this work must maintain attribution to the author(s) and the published article's title, journal citation, and DOI.
}

However, previous constructions of universal Hamiltonians have relied heavily on using perturbation gadgets, and constructing complicated "chains" of simulations to prove that simple models are indeed universal. For example, the original paper on universal quantum Hamiltonians used a chain of more than ten simulations and perturbationgadget constructions to prove universality of the twodimensional general Heisenberg and $X Y$ models. This work has recently been extended, with the first construction of a translationally invariant universal family of quantum Hamiltonians [3], again using a complex carefully engineered construction.

In Ref. [4], the present authors have developed a new method of proving universality and have used it to construct the first universal models acting in one dimension. This new method points to a connection between universality and complexity. This connection is not entirely surprising. Indeed, a rigorous complexitytheoretic characterization of universal Hamiltonians has already been demonstrated in the classical case [5]. Essentially, De las Cuevas and Cubitt [5] have shown that if a family of classical Hamiltonians has a ground-state energy problem that is NP hard, then it is necessarily also capable of simulating the complete physics of any other classical Hamiltonian. The converse implication is immediate. 
In the quantum setting, there have been hints that a similar result might hold. The classes of two-qubit interactions that are universal for simulating all, stoquastic, and classical Hamiltonians, respectively, have been fully characterized in Ref. [1] and have turned out to coincide precisely with the classes of interactions that have QMA-, StoqMA, and NP-complete ground-state energy problems. However, the proofs of these two classifications have been independent and it is certainly possible that this coincidence only applies in the case of qubits, as the proof techniques have relied critically on having only two-qubit interactions. Furthermore, the more complicated noncommutative structure of quantum Hamiltonians has made it impossible to replicate the classical approach of Ref. [1] to proving a relationship between complexity and simulation.

In this paper, by extending the simulation technique developed in Ref. [4], we resolve this. We derive necessary and sufficient complexity-theoretic conditions for a family of Hamiltonians to be an efficient universal model, relating this directly to complexity-theoretic properties of the ground state.

\section{MAIN RESULTS}

Our main result is a complexity-theoretic classification of which families of Hamiltonians are efficient universal models:

Theorem II.1 (Universality Classification): A family of Hamiltonians, $\mathscr{M}$, is an efficient universal model if and only if $\mathscr{M}$-HAMILTONIAN is QMA complete under faithful reductions and $\mathscr{M}$ is closed.

The first faithful reduction condition for a family of Hamiltonians $\mathscr{M}$ to be an efficient universal model is related to the complexity of $\mathscr{M}$-HAMILTONIAN - the problem of deciding whether or not a Hamiltonian in $\mathscr{M}$ has a low-energy ground state. We prove a connection between QMA completeness of $\mathscr{M}$-HAMILTONIAN and universality. However, QMA completeness alone is not enough for a model to be universal. We demonstrate that in order for $\mathscr{M}$ to be a universal model, $\mathscr{M}$-HAMILTONIAN must be QMA complete under faithful reductions, where we say that a reduction from a problem in QMA to $\mathscr{M}$-HAMILTONIAN is faithful if it maps the subspace picked out by a QMA -verification circuit to the low-energy subspace of the Hamiltonian - therefore preserving some of the structure of the verification circuit and the witness. (For a rigorous definition of faithfulness, see Sec. V.)

The second condition, closure, relates to combining different Hamiltonians from the same model. We say that a model, $\mathscr{M}$, is closed if, given $H_{A}^{(1)}, H_{B}^{(2)} \in \mathscr{M}$, acting on (possibly overlapping) sets of qudits $A, B$, respectively, there exists a Hamiltonian $H^{(3)} \in \mathscr{M}$ that can simulate $H_{A}^{(1)}+H_{B}^{(2)}$.
Furthermore, in this paper we provide a recipe for modifying history-state Hamiltonians so that the canonical reduction from a QMA problem to the history-state Hamiltonian is faithful. Therefore, all that remains to show that a family of history-state Hamiltonians is universal is to demonstrate closure.

We also derive two corollaries, giving complexitytheoretic conditions for families of Hamiltonians to be universal models that are not efficient in the sense of Ref. [1] but are nonetheless interesting. These corollaries, along with the main theorem, give a complete classification of all known universal models.

\section{DISCUSSION}

The most obvious implication of our results is that they provide a new route for proving universality of families of Hamiltonians. All previous universality results $[1,3,4,6]$ have relied on explicit constructions, tailored to particular universal models. These constructions have shown that given some arbitrary target Hamiltonian $H_{\text {target }}$, a Hamiltonian from the universal model can be constructed that simulates $H_{\text {target }}$. These constructions have typically drawn on techniques from Hamiltonian complexity theory, such as perturbation gadgets and history states, but the proofs of universality have required substantial additional work.

That additional work can now in many cases be sidestepped, by using the extensive existing work classifying the complexity of the local Hamiltonian problem [7-10], along with our main result (as well as our recipe for modifying history-state Hamiltonians). The remaining step is to demonstrate closure-for some families of Hamiltonians, this step is trivial (it follows from the definition of the model), while for others it will require some work - however, demonstrating closure will always be simpler than demonstrating universality, since closure requires the ability to simulate a very limited class of Hamiltonians.

It should be noted that this method of proving universality is not constructive, in the sense that it does not tell you how to simulate a given target Hamiltonian with a Hamiltonian from the a universal model. However, we do not view this as a drawback when compared to previous methods of proving universality. The previous methods for proving universality have been theoretically constructive - in the sense that for any target Hamiltonian, they have provided a mathematical description of a Hamiltonian from the universal model that could simulate it. However, in reality the cost of leaving the target Hamiltonian completely general in the previous proofs has been that the simulations constructed have had to be very complex - putting them out of reach of current experimental limitations. The benefit of our work is that it gives a simple route to proving universality, so that now the work on 
constructing explicit simulations can focus on simulations that are experimentally feasible.

The problem of constructing universal Hamiltonians where the simulations are experimentally feasible is challenging but solving it is of fundamental importance. Current constructions fall down in either requiring precise control of interaction strengths across many orders of magnitude or in requiring a very large local Hilbert-space dimension (and in some cases both). We believe that our results provide an extra tool for tackling this challenge. As already mentioned, our results give a new technique for proving universality. This could be applied to, for example, the construction in Ref. [11] of a low-dimensional spin chain with $\mathrm{QMA}_{\mathrm{EXP}}$-complete ground-state energy problem to check whether the model is universal. The construction in Ref. [11] is complex and checking universality via previous methods would be a difficult technical challenge. With the aid of our results, it becomes significantly more straightforward. If it is shown to be universal, it would be a universal model that does not require tuning of individual interaction strengths and with a local Hilbert-space dimension orders of magnitude smaller than any previously known model. This would be an important step toward experimentally feasible universal models.

The other direction in which our results could be used in is investigating the complexity of models that are currently used as analog simulators, to determine whether there is hope of using them to construct universal models. One platform that is currently used for analog Hamiltonian simulation is Rydberg atoms [12-14]. It has been suggested [12] that this might have promise as a universal simulator, as in certain regimes the platform naturally encodes the $X Y$ Hamiltonian, which is known to be universal [1]. However, the proof of universality cannot be used to construct a universal simulator using Rydberg atoms, as it involves complicated chains of perturbative simulations, requiring precise control beyond the reach of current experiments. An alternative approach to investigating the use of Rydberg atoms as universal simulators could be to investigate the complexity of the ground-state energy problem of Rydberg interactions where the control over interaction strengths is limited to what is experimentally feasible. As outlined above, demonstrating complexity in this regime is likely to be more straightforward than directly proving universality. If, within the limitations on interaction strength, it is possible to demonstrate hardness of the ground-state energy problem, that motivates attempts to look for simple universal constructions. If it is not possible to demonstrate hardness of the ground-state energy problem within current experimental limitations, it may be possible to determine how much experimental techniques have to advance in order to overcome the barrier and achieve universality.

Finally, the relationship between complexity and universality is interesting from a fundamental physics standpoint. It has already been clear that universality implies complexity - since universal models must be able to simulate all quantum many-body physics. However, the reverse direction has not been obvious. Our results show that if the problem of deciding whether a Hamiltonian in $\mathscr{M}$ has a low-energy ground state is hard for a quantum computer, then $\mathscr{M}$ must be rich enough to capture all quantum many-body physics.

The remainder of the paper contains the technical proofs of our main results. In Sec. IV, we cover some necessary technical background on universal Hamiltonians and complexity theory. The notion of a faithful reduction is outlined in detail in Sec. V; in this section, we also present a family of Hamiltonians that we demonstrate is QMA complete under faithful reductions. Finally, our main theorem is proved in Sec. VI.

\section{TECHNICAL PRELIMINARIES}

\section{A. Universal Hamiltonians}

In Ref. [1], a rigorous definition of what it means for one quantum system to simulate another has been developed:

Definition IV.1 (Approximate simulation [Def. 23] [1]): Let $\Delta, \eta, \epsilon>0$. A Hamiltonian $H^{\prime}$ is a $(\Delta, \eta, \epsilon)$ simulation of the Hamiltonian $H$ if there exists a local encoding $\mathscr{E}(M)=V(M \otimes P+\bar{M} \otimes Q) V^{\dagger}$ such that:

(i) There exists an encoding $\tilde{\mathscr{E}}(M)=\tilde{V}(M \otimes P+\bar{M} \otimes$ $Q) \tilde{V}^{\dagger}$ into the subspace $S_{\tilde{\mathscr{E}}}$ such that $S_{\tilde{\mathscr{E}}}=S_{\leq \Delta\left(H^{\prime}\right)}$ and $\|\tilde{V}-V\| \leq \eta$ and

(ii) $\left\|H_{\leq \Delta}^{\prime}-\tilde{\mathscr{E}}(H)\right\| \leq \epsilon$

where an encoding is a map of the form $\mathscr{E}(A)=$ $V(A \otimes P+\bar{A} \otimes Q) V^{\dagger}$ for $V$ an isometry and a local encoding maps local observables in the target system to local observables in the simulator system, defined as follows:

Definition IV.2 (Local subspace encoding (Definition 13 of Ref. [1])): Let

$$
\mathscr{E}: \mathscr{B}\left(\otimes_{j=1}^{N} \mathscr{H}_{j}\right) \rightarrow \mathscr{B}\left(\otimes_{j=1}^{N} \mathscr{H}_{j}\right),
$$

be a subspace encoding. We say that the encoding is local if for any operator $A_{j} \in \operatorname{Herm}\left(\mathscr{H}_{j}\right)$, there exists $A_{j}^{\prime} \in$ $\operatorname{Herm}\left(\mathscr{H}_{j}\right)$ such that

$$
\mathscr{E}\left(A_{j} \otimes \mathbb{1}\right)=\left(A_{j}^{\prime} \otimes \mathbb{1}\right) \mathscr{E}(\mathbb{1})
$$

Note that the role of $\tilde{\mathscr{E}}$ in Definition IV.1 is to provide an exact simulation as per [Def. 20][1]. However, it might not always be possible to construct this encoding in a local fashion. The local encoding $\mathscr{E}$ in turn approximates $\tilde{\mathscr{E}}$, such 
that the subspaces mapped to by the two encodings deviate by at most $\eta . \epsilon$ controls how much the eigenvalues are allowed to differ.

In Ref. [1], it is shown that approximate Hamiltonian simulation preserves important physical properties. We recollect the most important ones in the following:

Lemma IV.3 ([Lem. 27, Props. 28, 29][1]): Let $H$ act on $\left(\mathbb{C}^{d}\right)^{\otimes n}$. Let $H^{\prime}$ act on $\left(\mathbb{C}^{d^{\prime}}\right)^{\otimes m}$, such that $H^{\prime}$ is a $(\Delta, \eta, \epsilon)$ simulation of $H$ with corresponding local encoding $\mathscr{E}(M)=V(M \otimes P+\bar{M} \otimes Q) V^{\dagger}$. Let $p=\operatorname{rank}(P)$ and $q=\operatorname{rank}(Q)$. Then the following holds true:

(i) Denoting with $\lambda_{i}(H)$ [respectively, $\lambda_{i}\left(H^{\prime}\right)$ ] the $i$ thsmallest eigenvalue of $H$ (respectively, $\left.H^{\prime}\right)$, then, for all $1 \leq i \leq d^{N}$ and all $(i-1)(p+q) \leq j \leq i(p+$ q), $\left|\lambda_{i}(H)-\lambda_{j}\left(H^{\prime}\right)\right| \leq \epsilon$.

(ii) The relative error in the partition function evaluated at $\beta$ satisfies

$$
\begin{aligned}
& \frac{\left|\mathscr{Z}_{H^{\prime}}(\beta)-(p+q) \mathscr{Z}_{H}(\beta)\right|}{(p+q) \mathscr{Z}_{H}(\beta)} \\
& \quad \leq \frac{\left(d^{\prime}\right)^{m} e^{-\beta \Delta}}{(p+q) d^{N} e^{-\beta\|H\|}}+\left(e^{\epsilon \beta}-1\right) .
\end{aligned}
$$

(iii) For any density matrix $\rho^{\prime}$ in the encoded subspace for which $\mathscr{E}(\mathbb{1}) \rho^{\prime}=\rho^{\prime}$, we have

$$
\left\|e^{-i H^{\prime} t} \rho^{\prime} e^{i H^{\prime} t}-e^{-i \mathscr{E}(H) t} \rho^{\prime} e^{i \mathscr{E}(H) t}\right\|_{1} \leq 2 \epsilon t+4 \eta .
$$

If we are interested in whether an infinite family of Hamiltonians can be simulated by another, the notion of overhead becomes interesting: if the system size grows, how large is the overhead necessary for the simulation, in terms of the number of qudits, the operator norm, or the computational resources? We capture this notion in the following definition:

Definition IV.4 (Simulation, [Def. 23][1]): We say that a family $\mathscr{F}$ of Hamiltonians can simulate a family $\mathscr{F}$ of Hamiltonians if, for any $H \in \mathscr{F}$ and any $\eta, \epsilon>0$ and $\Delta \geq$ $\Delta_{0}$ (for some $\left.\Delta_{0}>0\right)$, there exists $H^{\prime} \in \mathscr{F}$ such that $\bar{H}^{\prime}$ is a $(\Delta, \eta, \epsilon)$ simulation of $H$.

We say that the simulation is efficient if, in addition, for $H$ acting on $n$ qudits and $H^{\prime}$ acting on $m$ qudits, $\left\|H^{\prime}\right\|=$ $\operatorname{poly}(n, 1 / \eta, 1 / \epsilon, \Delta)$ and $m=\operatorname{poly}(n, 1 / \eta, 1 / \epsilon, \Delta) ; H^{\prime}$ is efficiently computable given $H, \Delta, \eta$ and $\epsilon$; each local isometry $V_{i}$ in the decomposition of $V$ is itself a tensor product of isometries that map to $\mathrm{O}(1)$ qudits; and there is an efficiently constructable state $|\psi\rangle$ such that $P|\psi\rangle=|\psi\rangle$.

Definition IV.4 naturally leads to the question in which cases a family of Hamiltonians is so versatile that it can simulate any other Hamiltonian: in that case, we call the family universal:

Definition IV.5 (Universal Hamiltonians [Def. 26][1]): We say that a family of Hamiltonians is a universal simulator-or simply is universal - if any (finite-dimensional) Hamiltonian can be simulated by a Hamiltonian from the family. We say that the universal simulator is efficient if the simulation is efficient for all local Hamiltonians.

\section{B. Complexity theory}

Definition IV.6 $(\mathbf{Q M A}(\boldsymbol{c}, \boldsymbol{s})$ [10]): A promise problem $A=A_{Y E S} \cup A_{N O}$ is in $Q M A(c, s)$ if and only if there exists a polynomially bounded function $p$ and a quantum polynomial-time verifier $V$ such that for all $n$ and all $x \in\{0,1\}^{n}$ :

(a) If $x \in A_{Y E S}$, there exists a $p(n)$-qubit quantum state $|\psi\rangle$ such that $\operatorname{Pr}[V$ accepts $(x,|\psi\rangle)] \geq c$.

(b) If $x \in A_{N O}$ for all $p(n)$-qubit quantum states $|\psi\rangle$, $\operatorname{Pr}[\operatorname{Vaccepts}(x,|\psi\rangle)] \leq s$.

QMA $(c, s)$ where $c$ and $s$ are separated by an inverse polynomial is the class QMA [10]. QMA $(c, s)$ where $c$ and $s$ are separated by an exponentially small gap is the class PreciseQMA. PreciseQMA is known to be equal to the class PSPACE [15].

Definition IV.7 (QMA $\mathbf{Q X X P}_{\mathbf{E X}}(\boldsymbol{c}, \boldsymbol{s})$ [16]): A promise problem $A=A_{Y E S} \cup A_{N O}$ is in $Q M A_{\mathrm{EXP}}(c, s)$ if and only if there exists $a k$ and a quantum exponential time verifier $V$ such that, for all $n$ and all $x \in\{0,1\}^{n}$ :

(a) If $x \in A_{Y E S}$, there exists a $2^{n^{k}}$-qubit quantum state $|\psi\rangle$ such that $\operatorname{Pr}[V$ accepts $(x,|\psi\rangle)] \geq c$.

(b) If $x \in A_{N O}$ for all $2^{n^{k}}$-qubit quantum states $|\psi\rangle$, $\operatorname{Pr}[\operatorname{Vaccepts}(x,|\psi\rangle)] \leq s$.

$\mathrm{QMA}_{\mathrm{EXP}}(c, s)$ where $c$ and $s$ are separated by an inverse exponential is the class QMA $\mathrm{EXP}_{\text {. }}$

The canonical problem in Hamiltonian complexity is $k$ LOCAL HAMILTONIAN:

$k$-LOCAL HAMILTONIAN $(g)$

Input: Local Hamiltonian $H=\sum_{i=1}^{m} h_{i}$ on an $N$-partite Hilbert space of constant local dimension and $m \leq$ poly $N$. Each $h_{i}:=h_{S_{i}} \otimes \mathbb{1}_{S_{i}^{c}}$ acts nontrivially on at most $\left|S_{i}\right| \leq k$ sites and $\left\|h_{i}\right\| \leq 1$. Two numbers $\alpha, \beta>0$ with $\beta-\alpha \geq$ $g(N)$.

Promise: The ground-state energy $\lambda_{\min }(H)$ either $\geq \beta$ or $\leq \alpha$.

Question: YES if $\lambda_{\min }(H) \leq \alpha$, else NO. 
$k$-LOCAL HAMILTONIAN ( $1 /$ poly) is QMA complete for $k \geq 2$. $k$-LOCAL HAMILTONIAN ( $1 / \exp$ ) is PreciseQMA complete.

We can also consider the special case where the set of interaction terms and/or the geometry of the interaction graph is restricted (which can implicitly constrain the locality $k$ of the family):

Definition IV.8 ( $\mathscr{M}$-HAMILTONIAN): The $k$-LOCAL HAMILTONIAN (1/ poly) problem, where the Hamiltonian is restricted to belong to $\mathscr{M}$, some (possibly infinite) family of Hamiltonians.

Definition IV.9 (PRECISE- $\mathscr{M}$-HAMILTONIAN): The $k$ LOCAL HAMILTONIAN (1/ exp) problem, where the Hamiltonian is restricted to belong to $\mathscr{M}$, some (possibly infinite) family of Hamiltonians.

\section{FAITHFULNESS CONDITION}

\section{A. Faithful Hamiltonian reductions}

The acceptance operator, $Q(U)$, of a QMA-verification circuit, $U$, is defined as [Th. 3.6][17]:

$$
Q(U)=\left\langle\left. 0\right|^{\otimes m} U^{\dagger} \Pi_{\text {out }} U \mid 0\right\rangle^{\otimes m},
$$

where $U$ requires $m$ ancillas, initialized in the $|0\rangle$ state.

Definition V.1 (Gapped acceptance operators): Consider a promise problem A that can be verified by a unitary circuit $U$, with completeness probability c. Let $x \in A$, where $n=|x|$ is the size of the instance. Let $\lambda_{x}$ be the largest eigenvalue of $Q\left(U_{x}\right)$ that is less than the completeness $c$. We say that the acceptance operator is gapped if $c-\lambda_{x}>1 / \operatorname{poly}(n)$.

In other words, Definition V.1 means that any state with acceptance probability below the completeness threshold already lies significantly below it, namely $1 /$ poly bounded away.

Note that there is a subtle difference between the promise gap and the question of whether or not the acceptance operator is gapped. For any NO instance of a problem $A \in \mathrm{QMA}$, the definition of QMA trivially implies that the acceptance operator is gapped (since the acceptance probability is below the soundness threshold $\lambda_{x} \leq s$ and $c-s>$ $1 /$ poly by definition). However, for YES instances, it is possible to have an acceptance operator that is not gapped. We will see that for YES instances, the question of whether or not the acceptance operator is gapped is related to the spectral gap of a Hamiltonian, rather than the promise gap.

The idea of requiring a gap in the spectrum of proof systems has arisen before in the Hamiltonian complexity literature, first in Ref. [18] in the definition of the class PGQMA (polynomially gapped QMA) [19]. The notion of a gap in the spectrum of the proof system is again seen to be related to the spectral gap of a Hamiltonian, as $k$-LOCAL HAMILTONIAN with the added promise that the spectral gap of the Hamiltonian is inverse polynomial is complete for PGQMA. [18].

Definition V.2 (Faithful Hamiltonian reduction): Let $A=A_{Y E S} \cup A_{N O}$ be a promise problem that can be verified by a family of circuits, $U$, of length $T$, with completeness probability $c$. The acceptance operator $Q(U)$ is as defined in Eq. (3). Consider a reduction from $A$ to the $\mathscr{M}$-HAMILTONIAN problem. For a verification circuit with gapped $Q(U)$, we say that the reduction is faithful with respect to $U$ if, for all instances $x \in A_{Y E S}$, there exists a Hamiltonian $H_{x} \in \mathscr{M}$ acting on poly $(n)$ qudits (where $n=|x|)$ such that for the low-energy subspace,

$$
\mathscr{S}_{0}:=\operatorname{span}\left\{|\phi\rangle: H_{x}|\phi\rangle=\tilde{\lambda}|\phi\rangle, \tilde{\lambda} \leq \frac{\kappa(1-c)}{T+1}\right\}
$$

for $\kappa=1 / \operatorname{poly}(n)$, the following holds:

(i) $\left\|\Pi_{\mathscr{S}_{0}}-\Pi_{\mathscr{E}(\mathscr{L})}\right\| \leq \eta$, where

(a) $\eta<1$ can be made arbitrarily small.

(b) $\Pi$ denotes the projector onto the subspace $\mathscr{S}$.

(c) $\mathscr{E}$ is some local encoding (independent of the problem being encoded).

(d) $\mathscr{L}:=\operatorname{span}\{|\psi\rangle: Q(U)|\psi\rangle=\lambda|\psi\rangle, \lambda \geq c\}$.

(ii) The spectral gap above the subspace $\mathscr{S}_{0}$ is $\Omega[1 / \operatorname{poly}(T)]$.

For $x \in A_{N O}$, there are no conditions on $H_{x}$. Similarly, if $Q(U)$ is not gapped, there are no conditions on the $H_{x}$.

Note that the concept of a faithful reduction is a property of a particular verification circuit [20], not of the problem itself.

Definition V.3: We say that $\mathscr{M}$-HAMILTONIAN is $Q M A$ complete under faithful reductions if, for all $A \in Q M A$ and for any polynomial-time QMA-verification circuit $U$ that verifies $A$, there exists a reduction from $A$ to the $\mathscr{M}$-HAMILTONIAN problem that is faithful with respect to $U$.

\section{B. The modified Kitaev Hamiltonian}

The Hamiltonian that we use to prove necessity of the faithfulness condition is a modification of the 5-local Hamiltonian shown to be QMA complete in Ref. [10]. Note that this choice is convenient but the procedure we set out here to demonstrate faithfulness could be applied to any history-state Hamiltonian in the literature. 
The original 5-local Hamiltonian is a "circuit-toHamiltonian" mapping, given by

$$
H_{K}=H_{\text {in }}+H_{\text {prop }}+H_{\text {out }}+H_{\text {clock }},
$$

where the Hamiltonian is acting on the Hilbert space

$$
\begin{aligned}
\mathscr{H} & :=\mathscr{H}_{Q} \otimes \mathscr{H}_{C}=\left(\mathbb{C}^{2}\right)^{\otimes n} \otimes\left(\mathbb{C}^{2}\right)^{\otimes T+1} \\
& =\mathbb{C}^{2} \otimes\left(\mathbb{C}^{2}\right)^{\otimes|W|} \otimes\left(\mathbb{C}^{2}\right)^{\otimes|A|} \otimes\left(\mathbb{C}^{2}\right)^{\otimes T}
\end{aligned}
$$

and

$$
\begin{aligned}
H_{\text {in }} & =\Pi_{1}^{(1)} \otimes|0\rangle\left\langle\left. 0\right|_{1} ^{c}+\sum_{j \in A} \Pi_{j}^{(1)} \otimes \mid 0\right\rangle\left\langle\left. 0\right|_{1} ^{c}\right. \\
H_{\text {out }} & =\Pi_{1}^{(0)} \otimes|1\rangle\left\langle\left. 1\right|_{T} ^{c}\right. \\
H_{\text {clock }} & =\mathbb{1} \otimes \sum_{t+1}^{T-1}|01\rangle\left\langle\left. 01\right|_{t, t+1} ^{c}\right. \\
H_{\text {prop }} & =\frac{1}{2} \sum_{t=1}^{T-1} H_{t}
\end{aligned}
$$

with

$$
\begin{aligned}
H_{t}= & \mathbb{1} \otimes\left(|10\rangle\left\langle\left. 10\right|_{t, t+1} ^{c}+\mid 10\right\rangle\left\langle\left. 10\right|_{t+1, t+2} ^{c}\right)\right. \\
& -U_{t+1}|110\rangle\left\langle\left. 110\right|_{t-1, t, t+1} ^{c}-U_{t+1}^{\dagger} \mid 100\right\rangle\left\langle\left. 100\right|_{t-1, t, t+1} ^{c},\right.
\end{aligned}
$$

where the $U_{t}$ correspond to the gates applied at time $t$ in the circuit being encoded.

The Hamiltonian without the output penalty,

$$
H_{0}=H_{\text {in }}+H_{\text {prop }}+H_{\text {clock }},
$$

has a degenerate ground space spanned by states of the form

$$
\left|\eta^{(0, \alpha)}\right\rangle=\frac{1}{\sqrt{T+1}} \sum_{t=0}^{T}\left|\gamma_{t}^{(0, \alpha)}\right\rangle
$$

for arbitrary $\alpha$, where

$$
\left|\gamma_{t}^{(0, \alpha)}\right\rangle=\left|\alpha_{0}(t)\right\rangle \otimes\left|1^{t} 0^{T-t}\right\rangle^{c},
$$

in which $\left|\alpha_{0}(t)\right\rangle$ is the state of the quantum circuit at time $t$ if the input state of the ancillas and flag qubit correspond to the binary string $\mathbf{0}=0^{1+|A|}$ and the input state of the witness is given by $|\alpha\rangle$. by

The modified Kitaev Hamiltonian that we use is given

$$
H_{\mathrm{MK}}=H_{\text {in }}+H_{\text {prop }}+\kappa H_{\text {out }}+H_{\text {clock }} \text {, }
$$

where $\kappa=1 / \operatorname{poly}(T)=\mathrm{o}\left(1 / T^{3}\right)$.

\section{The $\mathscr{K}$-HAMILTONIAN problem is QMA complete under faithful reductions}

Let $\mathscr{K}$ be the family of Hamiltonians of the form given in Eq. (14). We begin by showing that $\mathscr{K}$-HAMILTONIAN is QMA complete and then show that we can always choose the reductions to be faithful.

Lemma V.4: $\mathscr{K}$-HAMILTONIAN is QMA complete.

Proof. The proof that $\mathscr{K}$-HAMILTONIAN is QMA complete is essentially unchanged from the proof of QMA completeness in Ref. [10]. We sketch the argument here very briefly. Assume that the circuit being encoded is a QMA-verification circuit with completeness parameter $c$ and soundness parameter $s$. First consider the YES instances. By definition, there exists a witness $w$ such that the verification circuit accepts with probability at least $c$. It follows that the ground state of $H_{\mathrm{MK}}$ has energy less than $\kappa(1-c) /(T+1)$.

For the NO cases, we use the following geometrical lemma.

Lemma V.5 (Geometrical lemma, Lemma 14.4 [10]): Let $H_{1}$ and $H_{2}$ be two Hamiltonians with ground energies $a_{1}$ and $a_{2}$, respectively. Suppose that for both Hamiltonians, the difference between the energy of the (possibly degenerate) ground space and the next-highest eigenvalue is larger than $\Lambda$ and that the angle between the two ground spaces is $\theta$. Then the ground energy of $\mathrm{H}_{1}+\mathrm{H}_{2}$ is at least $a_{1}+a_{2}+2 \Lambda \sin ^{2}(\theta / 2)$.

We apply Lemma V.5 to $H_{\mathrm{MK}}$ with $H_{1}=H_{\text {in }}+\kappa H_{\text {out }}$ and $H_{2}=H_{\text {prop }}+H_{\text {clock. }}$. We have $a_{1}=a_{2}=0$. The smallest nonzero eigenvalue of $H_{1}$ is $\kappa$ (since $H_{\text {in }}$ and $H_{\text {out }}$ are commuting projectors). The smallest nonzero eigenvalue of $H_{2}$ scales as $\Omega\left(1 / T^{2}\right)$ (see Ref. [10] for proof). The angle between the ground spaces satisfies

$$
\sin ^{2}(\theta / 2) \geq \frac{1-\sqrt{s}}{4(T+1)} .
$$

Again, the proof of this is unchanged from Ref. [10], as the ground space of $H_{1}$ is equal to the ground space of $H_{\text {in }}+H_{\text {out }}$.

Therefore, in NO instances, the ground energy of $H_{\mathrm{MK}}$ is lower bounded by $(1-\sqrt{s}) / \operatorname{poly}(T)$. Setting $c-s=$ $\Omega[1 / \operatorname{poly}(T)]$, we have $\beta-\alpha=\Omega[1 / \operatorname{poly}(T)]$. Therefore, we prove a reduction from QMA to the $k$-LOCAL HAMILTONIAN of $H_{\mathrm{MK}}$.

To show that we can always choose the reduction to be faithful, we first prove a lemma about the spectrum and low-energy subspace of $H_{\mathrm{MK}}$.

Lemma V.6: Consider a modified Kitaev Hamiltonian, $H_{M K}$, encoding the verification circuit of some QMA problem. Let $Q(U)$ be the acceptance operator for a verifier 
circuit $U$ for some $A \in Q M A$. Set

$$
\mathscr{C}_{0}:=\operatorname{span}\left\{\left|\eta^{(0, \phi)}\right\rangle: Q(U)|\phi\rangle=\lambda|\phi\rangle, \lambda>c\right\},
$$

where $c$ is the completeness parameter of the problem, and let $g:=c-\lambda_{x}$, where $\lambda_{x}$ is the largest eigenvalue of $Q(U)$ that is less than c, as in Definition V.1.

If $g>2 T^{3}(T+1) \kappa$, then there exists a unitary transformation $V$ such that the subspace $\mathscr{S}_{0}$ defined by $\Pi_{\mathscr{S}_{0}}:=$ $V^{\dagger} \Pi_{\mathscr{C}_{0}} V$ is the low-energy subspace of $H_{M K}$ :

$$
\mathscr{S}_{0}=\operatorname{span}\left\{|\psi\rangle: H_{M K}|\psi\rangle=\lambda|\psi\rangle, \lambda \leq \frac{\kappa(1-c)}{T+1}+T^{3} \kappa^{2}\right\}
$$$$
\left\|\Pi_{\mathscr{S}_{0}}-\Pi_{\mathscr{C}_{0}}\right\|=\mathrm{O}\left(T^{3} \kappa\right)
$$

and the spectral gap above $\mathscr{S}_{0}$ is given by $\Omega\{[g \kappa /(T+1)]$ $\left.-T^{3} \kappa^{2}\right\}$.

Proof. It is a standard result that the zero-energy groundstate subspace $\mathscr{G}$ of $H_{0}$ is spanned by history states $\left|\eta^{(0, \alpha)}\right\rangle$ for all $\alpha$. The spectral gap of $H_{0}$ is $\Omega\left(1 / T^{3}\right)$ [21].

Since $\left\|\kappa H_{\text {out }}\right\|=o\left(1 / T^{3}\right)<1 / 2 T^{3}$, the Hamiltonian $H_{\mathrm{MK}} \mid \mathcal{G}$ can be approximated by the Schrieffer-Wolff perturbative expansion (see the Appendix). Let $\Pi_{\mathscr{G}}$ be the projector onto $\mathscr{G}$.

The zeroth-order term in the expansion is given by $H_{0} \Pi_{\mathscr{G}}=0$. The matrix elements of the first-order term, $\Pi_{\mathscr{G}} H_{\text {out }} \Pi_{\mathscr{G}}$, are given by [22] [Appendix B]

$$
\left\langle\eta^{(0, \alpha)}\left|\Pi_{\mathscr{G}} H_{\mathrm{out}} \Pi_{\mathscr{G}}\right| \eta^{(0, \beta)}\right\rangle=\frac{\kappa}{T+1}(\langle\alpha|| \beta\rangle-\langle\alpha|Q| \beta\rangle) .
$$

Denote the eigenstates of $Q(U)$ by $\left|\phi_{1}\right\rangle,\left|\phi_{2}\right\rangle, \ldots,\left|\phi_{2^{w}}\right\rangle$ with associated eigenvalues $\lambda_{1} \geq \lambda_{2} \geq \cdots \geq \lambda_{2^{w}}$. In the basis spanned by $\left|\eta^{\left(0, \phi_{i}\right)}\right\rangle$, the first-order term in the Schrieffer-Wolff expansion is diagonal:

$$
\Pi_{\mathscr{G}} H_{\mathrm{out}} \Pi_{\mathscr{G}}=\frac{\kappa}{T+1} \sum_{i}\left(1-\lambda_{i}\right)\left|\eta^{\left(0, \phi_{i}\right)}\right\rangle\left\langle\eta^{\left(0, \phi_{i}\right)}\right| .
$$

By Eqs. (A12) and (19), we conclude that in $\mathscr{G}$ the eigenvalues of $H_{\mathrm{MK}}$ are given by

$$
\tilde{\lambda}_{i}=\frac{\kappa\left(1-\lambda_{i}\right)}{T+1} \pm T^{3} \kappa^{2}
$$

Let $\mathscr{R}:=\operatorname{span}\left\{|\psi\rangle: H_{\mathrm{MK}}|\psi\rangle=E|\psi\rangle, E \leq \kappa / 2\right\}$. By the $\sin (\theta)$ theorem [23],

$$
\left\|\Pi_{\mathscr{R}}-\Pi_{\mathscr{G}}\right\|=\left\|V^{\dagger} \Pi_{\mathscr{G}} V-\Pi_{\mathscr{G}}\right\| \leq T^{3} \kappa,
$$

where $V=e^{S}$ is the Schrieffer-Wolff transformation. Therefore,

$$
\left\|V^{\dagger} \Pi_{\mathscr{C}_{0}} V-\Pi_{\mathscr{C}_{0}}\right\|=\left\|\Pi_{\mathscr{S}_{0}}-\Pi_{\mathscr{C}_{0}}\right\| \leq T^{3} \kappa .
$$

$\mathscr{C}_{0}$ is spanned by history states satisfying $H_{\mathrm{eff}}(1)\left|\eta^{(0, \phi)}\right\rangle=$ $\lambda\left|\eta^{(0, \phi)}\right\rangle$ for $\lambda \leq \kappa(1-c) /(T+1)$. The corresponding eigenvalues of $H_{\mathrm{MK}}$ are upper bounded by $[\kappa(1-c) /$ $(T+1)]+T^{3} \kappa^{2}$. The smallest eigenvalue of $H_{\mathrm{MK}}$ that is larger than $[\kappa(1-c) /(T+1)]+T^{3} \kappa^{2}$ is lower bounded by $\left[\kappa\left(1-\lambda_{x}\right) /(T+1)\right]-T^{3} \kappa^{2}$.

Therefore, since $g>2 T^{3}(T+1) \kappa$, the subspace

$$
\mathscr{S}_{0}=\operatorname{span}\left\{|\psi\rangle: H_{\mathrm{MK}}|\psi\rangle=\lambda|\psi\rangle, \lambda \leq \frac{\kappa(1-c)}{T+1}+T^{3} \kappa^{2}\right\}
$$

and the spectral gap of $H_{\mathrm{MK}}$ above $\mathscr{S}_{0}$ is given by $\Omega\left(g \kappa /(T+1)-T^{3} \kappa^{2}\right)$.

Lemma V.7: The $\mathscr{K}$-HAMILTONIAN problem is QMA complete under faithful reductions.

Proof. For any verification circuit, $U$, of any problem in QMA, we can require that the computation "idles" in its initial state for $L$ time steps before carrying out its verification computation ("idling to enhance coherence" [21]).

The history state of the computation for the first $L$ time steps is given by

$$
\left|\eta_{\text {idling }}^{(0, \alpha)}\right\rangle=|\alpha\rangle \otimes|0\rangle^{|A|+1} \otimes \frac{1}{\sqrt{L}} \sum_{t=1}^{L}\left|1^{t} 0^{T-t}\right\rangle^{c} .
$$

The rest of the history state is captured in

$$
\left|\eta_{\text {comp }}^{(0, \alpha)}\right\rangle=\frac{1}{\sqrt{T-L}} \sum_{t=L+1}^{T}\left|\gamma_{t}^{(0, \alpha)}\right\rangle,
$$

so the total history state is given by

$$
\left|\eta^{(0, \alpha)}\right\rangle=\sqrt{\frac{L}{T}}\left|\eta_{\text {idling }}^{(0, \alpha)}\right\rangle+\sqrt{\frac{L-T}{T}}\left|\eta_{\text {comp }}^{(0, \alpha)}\right\rangle
$$

The encoding $\mathscr{E}(M)=V M V^{\dagger}$ defined via the isometry

$$
V=\sum_{i}\left|\eta_{\mathrm{idling}}^{(0, i)}\right\rangle\langle i|
$$

where the $|i\rangle$ are computational basis states, is local. (This can be verified by direct calculation, see Ref. [4].) 
Moreover, we have that

$$
\begin{aligned}
\left\|\Pi_{\mathscr{C}_{0}}-\Pi_{\mathscr{E}(\mathscr{L})}\right\|^{2} & =\left\|\sum_{|\phi\rangle \in \mathscr{L}}\left(\left|\eta^{(0, \phi)}\right\rangle\left\langle\eta^{0, \phi}|-| \eta_{\text {idling }}^{(0, \phi)}\right\rangle\left\langle\eta_{\text {idling }}^{(0, \phi)}\right|\right)\right\|^{2} \\
& =\left\|\sum_{|\phi\rangle \in \mathscr{L}}\left(\sqrt{\frac{T-L}{T}}\left|\eta_{\text {comp }}^{(0, \phi)}\right\rangle\left\langle\eta_{\text {comp }}^{(0, \phi)}\left|+\left(\sqrt{\frac{L}{T}}-1\right)\right| \eta_{\text {idling }}^{(0, \phi)}\right\rangle\left\langle\eta_{\text {idling }}^{(0, \phi)}\right|\right)\right\|^{2} \\
& \leq 2\left(1-\sqrt{\frac{L}{T}}\right)
\end{aligned}
$$

where $\mathscr{L}:=\{|\phi\rangle: Q|\phi\rangle=\lambda|\phi\rangle, \lambda>c\}$ Therefore $\| \Pi_{\mathscr{C}_{0}}$ $-\Pi_{\mathscr{E}(\mathscr{L})} \|$ can be made arbitrarily small by increasing $L$.

The result follows immediately from Lemma V.6 and the triangle inequality.

\section{GENERAL CONDITIONS FOR UNIVERSALITY}

In order to state our main theorem, we require one more definition.

Definition VI.1 (Closed Hamiltonian model): We say that a model $\mathscr{M}$ is closed if, for any pair of Hamiltonians $H_{A}^{(1)}, H_{B}^{(2)} \in \mathscr{M}$ acting on sets of qudits $A, B$, respectively, where in general $A \cap B \neq\{\}$, there exists a Hamiltonian $H^{(3)} \in \mathscr{M}$ that can efficiently simulate $H_{A}^{(1)}+H_{B}^{(2)}$.

We can now prove our main result, Theorem II.1.

Theorem II.1 (Universality Classification): A family of Hamiltonians, $\mathscr{M}$, is an efficient universal model if and only if $\mathscr{M}$-HAMILTONIAN is QMA complete under faithful reductions and $\mathscr{M}$ is closed.

Proof. First, consider the only-if direction. Closure is clearly necessary: if a model $\mathscr{M}$ is universal, all Hamiltonians (including those of the form $H_{A}^{(1)}+H_{B}^{(2)}$ for $\left.H_{A}^{(1)}, H_{B}^{(2)} \in \mathscr{M}\right)$ can be simulated by a Hamiltonian in the model. In Lemma V.7, we prove that the $\mathscr{K}$-HAMILTONIAN problem is QMA complete under faithful reductions. Any efficient universal model must be able to simulate Hamiltonians in $\mathscr{K}$ with only polynomial overhead; hence $\mathscr{M}$ HAMILTONIAN must itself be QMA complete under faithful reductions.

Now consider the if direction. Let $\mathscr{M}$ be a family of Hamiltonians meeting the conditions of the theorem, i.e., such that $\mathscr{M}$-HAMILTONIAN is QMA complete under faithful reductions, and $\mathscr{M}$ is closed. We explicitly construct a universal model, solely based on these conditions.
Consider the following problem:

YES-HAMILTONIAN

Input: A $k$-local Hamiltonian $H_{\text {target }}$ acting on $n$ spins with local dimension $d$.

Question: Output YES.

This problem is (clearly) trivial. But we can choose to construct a nontrivial QMA verification circuit for it. We choose a verification circuit that picks out a particular subspace that allows us to prove universality. By Definition V.3, there must be a faithful reduction with respect to this verification circuit from YES-HAMILTONIAN to $\mathscr{M}$-Hamiltonian.

The verification circuit that we choose, and the subspace it picks out, are captured in the following.

Lemma VI.2: YeS-HAMILTONIAN can be verified by a circuit $U_{a}$ with gapped acceptance operator $Q\left(U_{a}\right)$ with ground space

$$
\mathscr{L}_{0}:=\operatorname{span}\left\{|\phi\rangle: Q\left(U_{a}\right)|\phi\rangle=|\phi\rangle\right\}
$$

satisfying

$$
\left\|\Pi \mathscr{L}_{0}-\Pi_{\mathscr{W}}\right\| \leq \mathrm{O}\left(a^{-1}\right)
$$

where

$$
\begin{gathered}
\mathscr{W}:=\operatorname{span}\left\{\left|w_{\mu}\right\rangle=\frac{1}{\sqrt{a^{2}+1}}\left|\psi_{\mu}\right\rangle\left(a|\sigma\rangle+\left|E_{\mu}\right\rangle\right):\right. \\
\left.H_{\text {target }}\left|\psi_{\mu}\right\rangle=E_{\mu}\left|\psi_{\mu}\right\rangle\right\} .
\end{gathered}
$$

Proof. The verifier circuit, $C_{V}$, acts on the witness and two ancilla registers, $B, B^{\prime}$. It is helpful to divide the witness into two separate registers: an $A$ register, which is $n d$ dimensional qudits; and an $A^{\prime}$ register, which consists of $m$ qutrits with orthonormal basis states $|\sigma\rangle,|0\rangle$, and $|1\rangle$, 
where $m=\log _{2}(\epsilon)$. The $B$ register is the same size as the $A^{\prime}$ register. The $B^{\prime}$ register consists of a single qubit.

The verifier $C_{V}$ operates as follows:

(1) Apply a unitary rotation $P_{a}:|0\rangle \rightarrow\left(1 / \sqrt{a^{2}+1}\right)$ $(a|\sigma\rangle+|1\rangle)$ to the $B^{\prime}$ register.

(2) Carry out controlled-phase estimation on the $A$ register with respect to the unitary generated by $H_{\text {target }}$, $U=e^{i H_{\text {target }} \tau}$, for some $\tau$ such that $\left\|H_{\text {target }} \tau\right\|<2 \pi$.

The $B^{\prime}$ register serves as the control qubit. Calculate (an approximation to) the energy $E_{\mu}$ from the eigenphase $\theta_{\mu}$ and store the result in the $B$ register (in binary).

The calculation of $E_{\mu}$ to accuracy $\epsilon$ requires calculation of the eigenphase $\theta_{\mu}$ to accuracy $\mathrm{O}\left(\epsilon /\left\|H_{\text {target }}\right\|\right)$, which takes $\mathrm{O}\left(\left\|H_{\text {target }}\right\| / \epsilon\right)$ uses of $U=e^{i H_{\text {target }} \tau}$. The unitary $U$ must therefore be implemented to accuracy $O\left(\epsilon /\left\|H_{\text {target }}\right\|\right)$, which can be done with overhead $\operatorname{poly}\left(n, d^{k},\left\|H_{\text {target }}\right\|, \tau, 1 / \epsilon\right)$, where $n$ is the system size, $d$ is the local dimension, and $k$ is the locality via Lemma 3.3 of Ref. [4]. The whole procedure takes time $T_{\mathrm{PE}}=\operatorname{poly}\left(n, d^{k},\left\|H_{\text {target }}\right\| / \epsilon\right)$.

(3) Carry out a SWAP test [24] between registers $A^{\prime}$ and $B$. Accept if outcome 0 is measured; reject otherwise.

The entire procedure takes time $T=\mathrm{O}\left[\operatorname{poly}\left(n, d^{k}\right.\right.$, $\left.\left.\left\|H_{\text {target }}\right\|\right) / \epsilon\right]$.

Let

$$
\left|\alpha_{\mu}\right\rangle=\sum_{j}\left(\frac{1}{2^{m}} \sum_{k=0}^{2^{m}-1} e^{2 \pi i k\left(E_{\mu}-j / 2^{m}\right)}\right)|j\rangle
$$

be the result of applying the phase-estimation algorithm on $\left|\psi_{\mu}\right\rangle$ with respect to $U=e^{i H_{\text {target }} \tau}$. Then, evidently,

$$
\left|\phi_{\mu}\right\rangle=\frac{1}{\sqrt{a^{2}+1}}\left|\psi_{\mu}\right\rangle_{A}\left(a|\sigma\rangle_{A^{\prime}}+\left|\alpha_{\mu}\right\rangle_{A^{\prime}}\right)
$$

is an eigenvector of $Q\left(U_{a}\right)$ with eigenvalue 1, and all eigenvectors of $Q\left(U_{a}\right)$ with eigenvalue 1 are in $\operatorname{span}\left\{\left|\phi_{\mu}\right\rangle\right\}$.

Moreover,

$$
\left\langle w_{\mu}|| \phi_{\mu}\right\rangle \leq \frac{a^{2}+\left(4 / \pi^{2}\right)}{a^{2}+1} .
$$

Therefore,

$$
\|\left|w_{\mu}\right\rangle\left\langle w_{\mu}|-| \phi_{\mu}\right\rangle\left\langle\phi_{\mu} \|\right| \leq 2 \sqrt{1-\left(\frac{a^{2}+\left(4 / \pi^{2}\right)}{a^{2}+1}\right)^{2}}
$$

and

$$
\left\|\Pi_{\mathscr{L}_{0}}-\Pi_{\mathscr{W}}\right\| \leq \mathrm{O}\left(a^{-1}\right) .
$$

The next-largest eigenvalue of $Q\left(U_{a}\right)$ is $1 / 2$.
It follows immediately from Eq. (36) that

$$
\left\|\mathscr{E}\left(\Pi_{\mathscr{L}_{0}}\right)-\mathscr{E}\left(\Pi_{\mathscr{W}}\right)\right\| \leq \mathrm{O}\left(a^{-1}\right)
$$

for any encoding $\mathscr{E}$.

It follows from the triangle inequality and Definition V.2(i) that for any instance of YES-HAMILTONIAN there exists $H_{\mathrm{LS}} \in \mathscr{M}$ with low-energy subspace $\mathscr{S}_{0}:=$ $\operatorname{span}\left\{|\phi\rangle: H_{x}|\phi\rangle=\lambda_{\min }|\phi\rangle\right\}$ such that

$$
\left\|\Pi_{\mathscr{S}_{0}}-\Pi_{\mathscr{E}(\mathscr{W})}\right\| \leq \eta+\mathrm{O}\left(a^{-1}\right),
$$

where $\mathscr{E}=V(M \otimes P+\bar{M} \otimes Q) V^{\dagger}$ is some local encoding and $\eta$ can be chosen to be arbitrarily small. The spectral gap above $\mathscr{S}_{0}$ is $\Omega[1 / \operatorname{poly}(T)]$.

Another trivial problem (that is therefore also evidently in QMA) is the following:

FLAG IDENTIFICATION

Input: Classical description of a one-qudit state $|f\rangle$. Output: YES.

For this problem, we use a faithful reduction with respect to the nontrivial verification circuit that simply measures a single qudit in the $f$ basis. So, for any single-qudit state $|f\rangle$, there exists $H_{f} \in \mathscr{M}$ such that:

(a) $H_{f}\left|\mathscr{E}_{\text {state }}(\phi)\right\rangle=\lambda_{0}^{(f)}\left|\mathscr{E}_{\text {state }}(\phi)\right\rangle$ for all $|\phi\rangle$ such that $\langle\phi|| f\rangle=0$.

(b) $H_{f}\left|\mathscr{E}_{\text {state }}(f)\right\rangle=\lambda_{1}^{(f)}\left|\mathscr{E}_{\text {state }}(f)\right\rangle$.

for some local encoding $\mathscr{E}_{\text {state}}$, where $\lambda_{k}^{(f)}$ can be efficiently computed [since the problem size is $O(1)$ for a state $|f\rangle$ that can be described in $O(1)$ bits]. Without loss of generality, we take $\lambda_{k}^{(f)}=k$.

Consider a Hamiltonian acting on $N$ spins:

$$
H_{\text {sim }}=\Delta\left(H_{\mathrm{LS}}-\lambda_{\min } \mathbb{1}\right)+a \sum_{i=n^{\prime}+1}^{N} 2^{i-\left(n^{\prime}+1\right)} H_{i}^{(1)},
$$

where $H_{\mathrm{LS}} \in \mathscr{M}$ is a faithful reduction (with respect to the verifier defined in Lemma VI.2) from Yes-HAMILTONIAN for the Hamiltonian $H_{\text {target }}=\sum_{\mu} E_{\mu}\left|\psi_{\mu}\right\rangle\left\langle\psi_{\mu}\right| . E_{\mu}$ in $\left|w_{\mu}\right\rangle$ is expressed in binary to precision $\epsilon$ in qudits $\left[n^{\prime}+\right.$ 1,N] [25]. The $H_{i}^{(1)} \in \mathscr{M}$ are faithful reductions (with respect to the obvious verification circuit) from FLAG IDENTIFICATION for the flag states $|1\rangle$ acting on the $i$ th qudit. We require $\Delta>\left\|H_{\text {target }}\right\|$.

First, we show that $H_{\text {sim }}$ can simulate $H^{\prime}=\sum_{\mu} E_{\mu}\left|w_{\mu}\right\rangle$ $\left\langle w_{\mu}\right|$. The low-energy subspace of $H_{\mathrm{LS}}$ consists of states in the subspace $\mathscr{S}_{0}$. On states in $\mathscr{S}_{0}, H_{\mathrm{LS}}-\lambda_{\min }$ has energy zero; while on states in $\mathscr{E}(\mathscr{W}), a \sum_{i=n^{\prime}+1}^{N} 2^{i-\left(n^{\prime}+1\right)} H_{i}^{(1)}$ has energy in the range $\left[E_{\mu}-\epsilon, E_{\mu}+\epsilon\right]$. 
It follows from Lemma 24 of Ref. [1] and Eq. (38) that there exists an encoding $\mathscr{E}(M)=V^{\prime}(M \otimes P+\bar{M} \otimes Q)$ $V^{\prime \dagger}$ such that

$$
\left\|V^{\prime}-V\right\| \leq \sqrt{2}\left[\eta+O\left(a^{-1}\right)\right]
$$

and $\mathscr{E}(\mathbb{1})=\Pi \mathscr{S}_{0} \cdot$ Moreover,

$$
V^{\prime}=W V
$$

where $W$ is a unitary satisfying

$$
\Pi_{\mathscr{S}_{0}}=W \Pi_{\mathscr{E}(\mathscr{W})} W^{\dagger}
$$

and

$$
\|W-\mathbb{1}\| \leq \sqrt{2}\left\|\Pi_{\mathscr{S}_{0}}-\Pi_{\mathscr{E}(\mathscr{W}}\right\| \leq \mathrm{O}\left(\eta+a^{-1}\right) .
$$

We need the following technical lemma.

Lemma VI.3 (First-order simulation [Lemma 14][26]): Let $H_{0}$ and $H_{1}$ be Hamiltonians acting on the same space and let $\Pi$ be the projector onto the ground space of $H_{0}$. Suppose that $H_{0}$ is zero on $\Pi$ and that the next smallest eigenvalue is at least 1 . Let $U$ be an isometry such that $U U^{\dagger}=\Pi$ and

$$
\left\|U H_{\text {target }} U^{\dagger}-\Pi H_{1} \Pi\right\| \leq \epsilon / 2 .
$$

Let $H_{\text {sim }}=\Delta H_{0}+H_{1}$. Then there exists an isometry $\tilde{V}$ onto the space spanned by the eigenvectors of $H_{\mathrm{sim}}$ with eigenvalue less than $\Delta / 2$ such that:

(1) $\|U-\tilde{V}\| \leq \mathrm{O}\left(\Delta^{-1}\left\|H_{1}\right\|\right)$.

(2) $\left\|\tilde{V} H_{\text {target }} \tilde{V}^{\dagger}-H_{\text {sim }<\Delta / 2}\right\| \leq \mathrm{O}\left(\Delta^{-1}\left\|H_{1}\right\|^{2}\right)+\epsilon / 2$.

We apply Lemma VI.3 with $H_{1}=a \sum_{i=n^{\prime}+1}^{N} 2^{i-\left(n^{\prime}+1\right)}$ $H_{i}^{(1)}$ and $H_{0}=\delta H_{\mathrm{LS}}$, where $\delta=\mathrm{O}[\operatorname{poly}(T)]$. We have that in $\Pi_{\mathscr{S}_{0}}, H_{\mathrm{LS}}$ has energy zero and, by Definition V.2(ii), the spectral gap above $\mathscr{S}_{0}$ scales as $\Omega[1 / \operatorname{poly}(T)]$, so the next smallest eigenvalue of $H_{0}=\delta H_{\mathrm{LS}}$ is at least 1 .

Moreover, $\left\|H_{1}\right\|=a\left\|H_{\text {target }}\right\|$. Note that $V^{\prime}$ is an isometry that maps onto the ground state of $H_{0}, \mathscr{S}_{0}$. By construction, we have that the spectrum of $H_{\text {target }}$ is approximated to within $\epsilon$ by $H_{1}$ restricted to $\mathscr{E}(\mathscr{W})$, so $\| \Pi_{\mathscr{E}(\mathscr{W})} H_{1} \Pi_{\mathscr{E}(\mathscr{W}}-$ $\mathscr{E}\left(H_{\text {target }}\right) \| \leq \epsilon$.

Using that the operator norm is unitarily invariant, and that $V^{\prime}=W V$ gives

$$
\left\|W \Pi_{\mathscr{E}(\mathscr{W})} H_{1} \Pi_{\mathscr{E}(\mathscr{W})} W^{\dagger}-\mathscr{E}\left(H_{\text {target }}\right)\right\| \leq \epsilon .
$$

We also have

$$
\begin{aligned}
& \| \Pi \mathscr{S}_{0} H_{1} \Pi \mathscr{S}_{0}-W \Pi \mathscr{E}\left(\mathscr{W}_{1} H_{1} \Pi \mathscr{E}_{\mathscr{E}} W^{W^{\dagger}} \|\right. \\
& \quad=\left\|\Pi \mathscr{S}_{0} H_{1} \Pi \mathscr{S}_{0}-\Pi \mathscr{S}_{0} W H_{1} W^{\dagger} \Pi \mathscr{S}_{0}\right\| \\
& \quad \leq\left\|H_{1}-W H_{1} W^{\dagger}\right\| \\
& \quad \leq 2\left\|H_{1}\right\|\|\mathbb{1}-W\| \\
& \quad \leq \mathrm{O}\left(a \eta\left\|H_{\text {target }}\right\|\right),
\end{aligned}
$$

where we use Lemma 18 of Ref. [1] in the penultimate step. So

$$
\left\|\Pi \mathscr{S}_{0} H_{1} \Pi \mathscr{S}_{0}-\mathscr{E}\left(H_{\text {target }}\right)\right\| \leq \epsilon+\mathrm{O}\left(\text { a }\left\|H_{\text {target }}\right\|\right) .
$$

Lemma VI.3 therefore implies that there exists an isometry $\tilde{V}$ that maps exactly onto the low-energy space of $H_{\text {sim }}$ such that $\left\|\tilde{V}-V^{\prime}\right\| \leq \mathrm{O}\left(\left\|H_{\text {target }}\right\| a /(\Delta / \delta)\right)=$ $\mathrm{O}\left(a \delta\left\|H_{\text {target }}\right\| / \Delta\right)$. By the triangle inequality and Eq. (40), we have

$$
\begin{aligned}
\|V-\tilde{V}\| & \leq\left\|V-V^{\prime}\right\|+\left\|V^{\prime}-\tilde{V}\right\| \\
& \leq \mathrm{O}\left(\frac{a \operatorname{poly}\left(T^{\prime}\right)\left\|H_{\text {target }}\right\|}{\Delta}+\eta+a^{-1}\right) .
\end{aligned}
$$

The second part of the lemma implies that

$$
\begin{aligned}
& \left\|\tilde{V} H^{\prime} \tilde{V}^{\dagger}-\left(H_{\text {sim }}\right)_{<\Delta^{\prime} / 2}\right\| \\
& \quad \leq \epsilon+\mathrm{O}\left(a \eta\left\|H_{\text {target }}\right\|+\left(a \mid H_{\text {target }} \|\right)^{2} /(\Delta / \delta)\right) \\
& =\epsilon+\mathrm{O}\left(a \eta\left\|H_{\text {target }}\right\|+\frac{a^{2} \mid H_{\text {target }} \|^{2} \delta}{\Delta}\right) .
\end{aligned}
$$

Therefore, the conditions of Definition IV.1 are satisfied for a $\left(\Delta^{\prime}, \eta^{\prime}, \epsilon^{\prime}\right)$ simulation of $H^{\prime}$, with $\eta^{\prime}=$ $\mathrm{O}\left\{\left[a\right.\right.$ poly $\left.\left.\left(T^{\prime}\right)\left\|H_{\text {target }}\right\| / \Delta\right]+\eta+a^{-1}\right\}, \quad \epsilon^{\prime}=\epsilon+\mathrm{O}\{a \eta$ $\left.\left\|H_{\text {target }}\right\|+\left[a^{2} \operatorname{poly}\left(T^{\prime}\right)\left\|H_{\text {target }}\right\|^{2} / \Delta\right]\right\}$ and $\Delta^{\prime}=\Delta / \delta=$ $\Delta / \operatorname{poly}(T)$.

By definition, we can choose $\eta$ to be arbitrarily small. We can also make $\mathrm{O}\left(a^{-1}\right)$ arbitrarily small. By increasing $T$, we can also make $\epsilon$ arbitrarily small. Therefore, by choosing $\Delta$ such that

$$
\begin{aligned}
\Delta \geq & \Delta^{\prime} \operatorname{poly}\left(T^{\prime}\right)+\frac{a \operatorname{poly}\left(T^{\prime}\right)\left\|H_{\text {target }}\right\|}{\eta^{\prime}} \\
& +\frac{a^{2} \operatorname{poly}\left(T^{\prime}\right)\left\|H_{\text {target }}\right\|^{2}}{\epsilon^{\prime}},
\end{aligned}
$$

we can construct $H_{\text {sim }}$, which is a $\left(\Delta^{\prime}, \eta^{\prime}, \epsilon^{\prime}\right)$ simulation of $H^{\prime}$ with arbitrarily small $\epsilon^{\prime}, \eta^{\prime}$. Since $H_{\text {sim }}$ is a sum of Hamiltonians that are all in $\mathscr{M}$, by the closure property there exists $H_{\text {univ }} \in \mathscr{M}$ that can efficiently simulate $H_{\text {sim }}$. Therefore, since simulations compose (which is proved in ref. [1], Lemma 17) we have that $H_{\text {univ }}$ can simulate $H^{\prime}$. 
Finally, we show that $H^{\prime}=\sum_{\mu} E_{\mu}\left|w_{\mu}\right\rangle\langle$ is itself a simulation of $H_{\text {target }}$. Consider the local encoding

$$
\mathscr{E}(M)=W M W^{\dagger},
$$

where $W=\sum_{\mu}\left|\psi_{\mu}\right\rangle|0\rangle\left\langle\psi_{\mu}\right|$, and the nonlocal encoding

$$
\tilde{\mathscr{E}}(M)=\tilde{W} M \tilde{W}^{\dagger},
$$

with

$$
\tilde{W}=\frac{1}{\sqrt{a^{2}+1}} \sum_{\mu}\left|\psi_{\mu}\right\rangle\left(a|\sigma\rangle+\left|E_{\mu}\right\rangle\right)\left\langle\psi_{\mu}\right| .
$$

We have that

$$
\|W-\tilde{W}\|=2\left(1-\frac{a}{\sqrt{a^{2}+1}}\right),
$$

so by increasing $a$ we can make the norm arbitrarily small. We also have that $S_{\tilde{\mathscr{E}}_{\prime}}=S_{H^{\prime}}$, so condition (i) from Definition IV.1 is met. The spectrum of $H^{\prime}$ is exactly the spectrum of $\tilde{\mathscr{E}}^{\prime}\left(H_{\text {target }}\right)$, so condition (ii) of Definition IV.1 is also met. Therefore, $H^{\prime}$ is a simulation of $H_{\text {target }}$.

Using the composition of simulations again, we have that $H_{\text {univ }}$ can simulate $H_{\text {target }}$. We have left $H_{\text {target }}$ arbitrary, so $\mathscr{M}$ is a universal model.

Finally, we consider efficiency. The simulation of $H_{\text {target }}$ by $H^{\prime}$ is clearly efficient. To see that the simulation of $H^{\prime}$ by $H_{\text {sim }}$ is efficient, note that the number of qudits in the simulation, $N$, must be polynomial in $n$ and $\left\|H_{\text {target }}\right\|$ as $H_{\mathrm{LS}}$ is in QMA. Furthermore, $\left\|H_{\text {sim }}\right\|=\Omega(\Delta)=\operatorname{poly}\left(T^{\prime},\left\|H_{\text {target }}\right\|, 1 / \epsilon^{\prime}, 1 / \eta^{\prime}\right)=$ $\operatorname{poly}\left(n,\left\|H_{\text {target }}\right\|, 1 / \epsilon^{\prime}, 1 / \eta^{\prime}\right)$. Thus $H_{\text {sim }}$ is an efficient simulation of $H^{\prime}$.

There are two corollaries about universal Hamiltonians that are not efficient in the sense of Ref. [1] but that are nonetheless interesting universal models that are better suited to some applications.

Definition VI.4: We say that a Hamiltonian can be described succinctly if it can be described by $\mathrm{O}[\log (n)]$ bits of information when acting on $n$ qudits.

Corollary VI.5: Let $\mathscr{M}$ be a family of succinct Hamiltonians. Then $\mathscr{M}$ is universal and can efficiently simulate any succinct Hamiltonian if and only if $\mathscr{M}$-HAMILTONIAN is $Q M A_{\mathrm{EXP}}$ complete under faithful reductions and $\mathscr{M}$ is closed.

Proof. This proof relies on the same ideas as Theorem II.1, so here we sketch the main ideas, highlighting where the proofs differ. First, note that the proof that
$\mathscr{K}$-HAMILTONIAN is QMA complete under faithful reductions (Lemma V.7) can be repurposed to prove that the $\mathscr{M}$-HAMILTONIAN which has been shown to be $\mathrm{QMA}_{\text {EXP }}$ complete in Ref. [8] remains $\mathrm{QMA}_{\mathrm{EXP}}$ complete under faithful reductions. The only-if direction follows immediately as in Theorem II.1 (where now the overhead can be exponential).

To see the if direction, we assume that there exists a family of Hamiltonians, $\mathscr{M}$, such that $\mathscr{M}$-HAMILTONIAN is $\mathrm{QMA}_{\mathrm{EXP}}$ complete under faithful reductions and $\mathscr{M}$ is closed. Consider the following computational problem:

SUCCINCT-YES-HAMILTONIAN

Input: A $k$-local Hamiltonian $H_{\text {target }}$ acting on $n$ spins with local dimension $d$, which can be described succinctly

Question: Output YES

SUCCINCT-YeS-HAMILTONIAN is clearly a trivial problem. But, as with Yes-HAMILTONIAN, we can construct a nontrivial verification circuit, which picks out a particular subspace that allows us to prove universality. By Definition V.3, there must be a faithful reduction with respect to this verification circuit from SUCCINCT-YES-HAMILTONIAN to $\mathscr{M}$-HAMiLTONIAN.

The verification circuit that we choose, and the subspace it picks out, are as in Lemma VI.2. Although the circuit is unchanged, this is now a $\mathrm{QMA}_{\mathrm{EXP}}$-verification circuit, since for Hamiltonians acting on $n$ qudits, the circuit length and witness size are of order $\operatorname{poly}(n)=\mathrm{O}\left(2^{\operatorname{poly}(x)}\right)$, where $x$ is the number of bits of information needed to describe the input to the problem.

It then follows, using the same argument as in Lemma VI.2, that for any Hamiltonian $H_{\text {target }}$ acting on $n$ spins that can be described succinctly, there exists a Hamiltonian in $\mathscr{M}$ that can simulate $H_{\text {target }}$ efficiently (where efficiency is defined in terms of the number of qudits, not bits of information).

To prove universality, note that in Theorem 3.6 of Ref. [4], a construction is given of a universal Hamiltonian, $H_{\text {succ }}$ (with an exponential overhead in terms of the number of spins and the norm of the simulating system), which can be described succinctly.

Since there exists a Hamiltonian in $\mathscr{M}$ that can simulate $H_{\text {succ }}$ (for any values of the parameters in $H_{\text {succ }}$ ) and since simulations compose, it follows that $\mathscr{M}$ is a universal model. When simulating general (nonsuccinct) Hamiltonians, the universal model, $\mathscr{M}$, inherits an exponential overhead in terms of the numbers of qudits and the norm of the simulating system from $H_{\text {succ }}$.

$\mathrm{QMA}_{\mathrm{EXP}}$ is a more powerful complexity class than QMA, so it may seem odd that it appears to be less efficient as a simulator. However, there are some situations where using a family of Hamiltonians meeting the conditions of Corollary VI.5 will give a more efficient simulator 
than using a family of Hamiltonians meeting the conditions of Theorem II.1. To see this, note that given a Hamiltonian that can be described succinctly, it can be simulated efficiently (in the sense of Ref. [1], i.e., in terms of numbers of qudits, simulating the system norm, and $\epsilon$ and $\eta$ parameters) by either a family of Hamiltonians with a QMAcomplete $\mathscr{M}$-HAMILTONIAN or a family of Hamiltonians with a QMAEXP-complete $\mathscr{M}$-HAMILTONIAN. However, the simulation by the QMA-complete family of Hamiltonians will not be efficient in terms of the number of bits needed to describe the simulating Hamiltonian, whereas

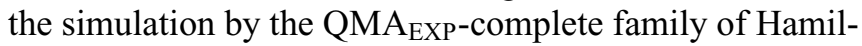
tonians would be. So there are situations where simulation using a QMAEXP-complete family of Hamiltonians is more efficient, demonstrating that the question of which family of Hamiltonians is a "more powerful" simulator does not have a straightforward answer.

The obvious example of Hamiltonians that can be succinctly described is the family of translationally invariant Hamiltonians. Examples of translationally invariant universal Hamiltonians are constructed in Ref. [3,4], where it is noted that a translationally invariant universal model with fixed interactions must have an exponential overhead in terms of the number of spins by a simple counting argument.

By considering the problem PRECISE- $\mathscr{M}$-HAMILTONIAN and introducing the idea of a exponentially faithful reduction, we can also derive conditions for universal models that are efficient in terms of the number of qudits in the simulator system, but not in terms of the norm of the simulating system. We say that a reduction is exponentially faithful if it meets the conditions of Definition V.2, but where now the gap in the spectrum of the acceptance operator, $g$, and the corresponding gap in the spectrum of the Hamiltonian are required to satisfy $g>1 / \exp (n)$ for $n$ the size of the input. This is a natural relaxation when considering PRECISE- $\mathscr{M}$-HAMiltonian, as it requires the gap in the spectrum of the acceptance operator in YES cases to scale in the same way as the promise gap for the problem.

The natural complexity class when considering PRECISE$\mathscr{M}$-HAmiltonian is PreciseQMA. It is known that every problem in PreciseQMA can be solved by a quantum circuit of length $T=\mathrm{O}[\exp (n)]$ acting on $\operatorname{poly}(n)$ qudits, with completeness $c=1-2^{\text {poly(n) }}$ and soundness $s=2^{\text {poly }(n)}$ (where $n$ is the size of the problem input) [Corollary 10][15]. Therefore, when defining what it means for PRECiSE- $\mathscr{M}$-HAMILTONIAN to be PreciseQMA complete under exponentially faithful reductions, there are two classes of circuits with respect to which we could require faithfulness - the polynomial-sized circuits that give an exponentially small completeness-soundness gap or the exponential-sized circuits that give a completenesssoundness gap exponentially close to 1 . Here, we choose the latter and define the following.
Definition VI.6: We say that PRECISE- $\mathscr{M}$-HAMILTONIAN is PreciseQMA complete under exponentially faithful reductions if, for all $A \in$ Precise $Q M A$ and for any exponential time verification circuit $U$ that verifies $A$, there exists a reduction from $A$ to the PRECISE- $\mathscr{M}$-HAMILTONIAN problem that is exponentially faithful with respect to $U$.

Corollary VI.7: A family of Hamiltonians, $\mathscr{M}$, is a universal model that is:

(1) Efficient in terms of the numbers of qudits

(2) Not efficient in terms of the norm of the simulating system and

(3) Achieves exponential accuracy in the $\epsilon$ parameter with polynomial overhead in number of qudits and exponential overhead in simulating system norm

if and only if

(i) Precise- $\mathscr{M}$-Hamiltonian is PSPACE complete under exponentially faithful reductions

(ii) $\mathscr{M}$ is closed and

(iii) $\mathscr{M}$-HAMILTONIAN is not QMA complete under faithful reductions

Proof. Recall that PSPACE $=$ PreciseQMA [15].

First, consider the if direction. Assume that we have a family of Hamiltonians $\mathscr{M}$ meeting the conditions $i-$ iii of the theorem. Since $\mathscr{M}$-HAMILTONIAN is not QMA complete under faithful reductions, we have to use exponentially faithful reductions to PRECISE- $\mathscr{M}$-HAMILTONIAN, where by Definition VI.6 we are considering faithfulness with respect to exponential time circuits. The if direction follows immediately, since going through the proof of Theorem II.1 with an exponentially small gap in the acceptance operator and exponentially long computation time requires an exponentially large energy penalty $\Delta$ and gives an exponentially small accuracy parameter $\epsilon$.

To see the only-if direction, consider a universal model, $\mathscr{M}$ meeting conditions $1-3$ of the theorem. Necessity of closure is trivial. Note that $\mathscr{M}$-HAMILTONIAN cannot be QMA complete under faithful reductions, because by Theorem II.1, if it was, the universal model would be efficient in terms of norm.

Consider simulating the family of Hamiltonians $\mathscr{K}$ using the model $\mathscr{M}$ to exponential accuracy in $\epsilon$. This demonstrates that PRECISE- $\mathscr{M}$-HAMILTONIAN is PSPACE complete (including under exponentially faithful reductions) but it does not contradict the statement that $\mathscr{M}$ HAMILTONIAN is not QMA complete. This is because the $k$-LOCAL HAMILTONIAN problem requires that the terms in the Hamiltonian are of order 1, which requires dividing each term in the simulator system by the norm of the simulating system, which by assumption is exponential in the size of the system. This leads to a Hamiltonian with 
an exponentially small spectral gap, which attenuates the promise gap too fast to maintain QMA completeness but gives PreciseQMA completeness (and therefore PSPACE completeness).

An example of a universal Hamiltonian meeting the conditions of Corollary VI.7 is given in Ref. [4]. It is a translationally invariant universal model but it includes a phase parameter that encodes information about the target system, so the interactions are not fixed.

\section{ACKNOWLEDGMENTS}

J.B. acknowledges support from the Draper's Junior Research Fellowship at Pembroke College. T.S.C. is supported by the Royal Society. T.K. is supported by the Engineering and Physical Sciences Research Council (EPSRC) Centre for Doctoral Training in Delivering Quantum Technologies, under Grant No. EP/L015242/1. This work was supported by the EPSRC Prosperity Partnership in Quantum Software for Simulation and Modelling, under Grant No. EP/S005021/1.

\section{APPENDIX: THE SCHRIEFFER-WOLFF EXPANSION}

Consider a finite-dimensional Hilbert space decomposed into a direct sum:

$$
\mathscr{H}=\mathscr{H}_{+} \oplus \mathscr{H}_{-} .
$$

Let $\Pi_{-}$be the projector onto $\mathscr{H}_{-}$and let $\Pi_{+}$be the projector onto $\mathscr{H}_{+}$. Let $H_{0}$ and $H_{1}$ be Hermitian operators acting on $\mathscr{H}$ such that $H_{0}$ is block diagonal with respect to the direct sum. Assume that all the eigenvalues of $H_{0}$ on $\mathscr{H}_{-}$ are in the range $\left[0, \lambda_{0}\right]$ for $\lambda_{0}<1$.

Consider the perturbed Hamiltonian $\tilde{H}=\Delta H_{0}+H_{1}$, where $\Delta \gg 1$ and $\|V\|<\Delta / 2$. The Schrieffer-Wolf transformation is a unitary rotation $e^{S}$, which is used to perturbatively diagonalize $\tilde{H}$. It satisfies the following properties:

$$
\begin{gathered}
\Pi_{-}\left(e^{S} \tilde{H} e^{-S}\right) \Pi_{+}=\Pi_{+}\left(e^{S} \tilde{H} e^{-S}\right) \Pi_{-}=0, \\
\Pi_{-} S \Pi_{-}=\Pi_{+} S \Pi_{+}=0, \quad\|S\|<\frac{\pi}{2} .
\end{gathered}
$$

The effective low-energy Hamiltonian $H_{\text {eff }}$ acting on $\mathscr{H}_{-}$ is given by

$$
H_{\mathrm{eff}}=\Pi_{-}\left(e^{S} \tilde{H} e^{-S}\right) \Pi_{-} .
$$

Define $\mathscr{R}:=\left\{|\psi\rangle:\langle\psi|\tilde{H}| \psi\rangle \in\left[\lambda_{0}-(\Delta / 2), \lambda_{0}+(\Delta / 2)\right]\right\}$ and let $\Pi_{\mathscr{R}}$ be the projector onto $\mathscr{R}$. Then,

$$
e^{S} \Pi_{\mathscr{R}} e^{-S}=\Pi_{-} .
$$

The operators $S$ and $H_{\text {eff }}$ can be expressed as Taylor series:

$$
\begin{aligned}
& S=\sum_{j=1}^{\infty} S_{j} \\
& H_{\mathrm{eff}}=\sum_{j=1}^{\infty} H_{\mathrm{eff}}^{(j)} .
\end{aligned}
$$

A systematic method for calculating the Taylor coefficients is given in Section 3.2 of Ref. [27]. We only need the first two coefficients:

$$
H_{\text {eff }}^{(0)}=H_{0} \Pi_{-} \quad \text { and } \quad H_{\text {eff }}^{(1)}=\Pi_{-} H_{1} \Pi_{-} .
$$

The size of the operators in the Taylor expansion can be bounded (see Lemma 3.4 of Ref. [27]):

$$
\begin{aligned}
& \left\|S_{j}\right\| \leq \mathrm{O}\left[\left(1+\frac{\lambda_{0}}{\pi \Delta}\right)\left(\frac{\left\|H_{1}\right\|}{\Delta}\right)^{j}\right] \\
& \left\|H_{\mathrm{eff}}^{(j)}\right\| \leq \mathrm{O}\left[\Delta\left(1+\frac{\lambda_{0}}{\pi \Delta}\right)\left(\frac{\left\|H_{1}\right\|}{\Delta}\right)^{j}\right] .
\end{aligned}
$$

This implies [26] that

$$
\begin{aligned}
& \|S\| \leq \mathrm{O}\left[\Delta^{-1}\left\|H_{1}\right\|\left(1+\frac{\lambda_{0}}{\pi \Delta}\right)\right] \\
& \left\|H_{\text {eff }}-H_{\text {eff }}(k)\right\| \leq \mathrm{O}\left[\Delta^{-k}\left\|H_{1}\right\|^{k+1}\left(1+\frac{\lambda_{0}}{\pi \Delta}\right)\right]
\end{aligned}
$$

where $H_{\mathrm{eff}}(k)=\sum_{j=1}^{k} H_{\mathrm{eff}}^{(j)}$.

[1] T. Cubitt, A. Montanaro, and S. Piddock, Universal quantum Hamiltonians, Proc. Natl. Acad. Sci. 115, 9497 (2018).

[2] T. Kohler and T. Cubitt, Toy models of holographic duality between local Hamiltonians, J. High Energy Phys. 2019, 17 (2019).

[3] S. Piddock and J. Bausch, Universal translationallyinvariant Hamiltonians, ArXiv:2001.08050 (2020).

[4] T. Kohler, S. Piddock, J. Bausch, and T. Cubitt, Translationally-invariant universal quantum Hamiltonians in 1D, ArXiv:2003.13753 (2020).

[5] G. De las Cuevas and T. S. Cubitt, Simple universal models capture all classical spin physics, Science 351, 1180 (2016).

[6] L. Zhou and D. Aharonov, Strongly universal Hamiltonian simulators, ArXiv:2102.02991 (2021).

[7] T. S. Cubitt and A. Montanaro, in 2014 IEEE 55th Annual Symposium on Foundations of Computer Science (IEEE, New York, 2014), p. 120.

[8] D. Gottesman and S. Irani, The quantum and classical complexity of translationally invariant tiling and Hamiltonian problems, Theory Comput. 9, 31 (2009). 
[9] J. Kempe and O. Regev, 3-local Hamiltonian is QMAcomplete, Quantum Inf. Comput. 3, 258 (2003).

[10] A. Y. Kitaev, A. Shen, and M. N. Vyalyi, in Quantum Information (Springer New York, New York, 2002), p. 203.

[11] J. Bausch and S. Piddock, The complexity of translationally invariant low-dimensional spin lattices in 3D, J. Math. Phys. 58, 111901 (2017).

[12] M. Morgado and S. Whitlock, Quantum simulation and computing with Rydberg-interacting qubits, AVS Quantum Sci. 3, 023501 (2021).

[13] P. Scholl, M. Schuler, H. J. Williams, A. A. Eberharter, D. Barredo, K.-N. Schymik, V. Lienhard, L.-P. Henry, T. C. Lang, T. Lahaye, et al. Quantum simulation of 2D antiferromagnets with hundreds of Rydberg atoms, Nature 595, 233 (2021).

[14] X. Wu, X. Liang, Y. Tian, F. Yang, C. Chen, Y.-C. Liu, M. K. Tey, and L. You, A concise review of Rydberg atom based quantum computation and quantum simulation*, Chin. Phys. B 30, 020305 (2021).

[15] B. Fefferman and C. Y.-Y. Lin, in Leibniz International Proceedings in Informatics (LIPICS). 9th Innovations in Theoretical Computer Science Conference (2016).

[16] D. Gottesman and S. Irani, The quantum and classical complexity of translationally invariant tiling and Hamiltonian problems, ArXiv:0905.2419 (2009).

[17] C. Marriott and J. Watrous, Quantum Arthur-Merlin games, (Dagstuhl, Saarbrücken/Wadern, Germany, 2005).

[18] D. Aharonov, M. Ben-Or, F. G. S. L. Brandao, and O. Sattath, The pursuit for uniqueness: Extending Valiant-Vazirani theorem to the probabilistic and quantum settings, ArXiv:0810.4840 (2008).
[19] PGQMA is a similar class to QMA with the added condition that the acceptance operator of the verification circuit has an inverse polynomial spectral gap. Note that for PGQMA, the gap is required to be between the lowest and second-lowest eigenvalues, unlike in our definition.

[20] Although any equivalent model of computation could be substituted into the definition.

[21] D. Aharonov, W. van Dam, J. Kempe, Z. Landau, S. Lloyd, and O. Regev, Adiabatic quantum computation is equivalent to standard quantum computation, SIAM J. Comput. 37, 166 (2007).

[22] A. Deshpande, A. V. Gorshkov, and B. Fefferman, The importance of the spectral gap in estimating ground-state energies, ArXiv:2007.11582 (2020).

[23] C. Davis and W. M. Kahan, Some new bounds on perturbation of subspaces, Bull. Am. Math. Soc. 75, 863 (1969).

[24] H. Buhrman, R. Cleve, J. Watrous, and R. de Wolf, Quantum Fingerprinting, Phys. Rev. Lett. 87, 167902 (2001).

[25] Here, $n^{\prime}$ is the number of spins in the encoded witness state $\mathscr{E}\left(\left|w_{\mu}\right\rangle\right)$. Since this is a reduction to QMA, we have $n^{\prime}=$ $\mathrm{O}(\operatorname{poly}(n))$.

[26] S. Bravyi and M. Hastings, On complexity of the quantum Ising model, Commun. Math. Phys. 349, 1 (2017).

[27] S. Bravyi, D. P. DiVincenzo, and D. Loss, SchriefferWolff transformation for quantum many-body systems, Ann. Phys. (N.Y.) 326, 2793 (2011).

Correction: The article identification number was assigned incorrectly during the final production stages and has been fixed. 